\title{
A Coupled Relaxation Method for Finding Perceptual Structures
}

\author{
Richard C. Wilson \\ Dept. of Computer Science \\ University of York, UK
}

\begin{abstract}
In this paper we describe a method for determining the existence and parameters of a geometric structure in an image by using a relational representation of its structure. The relational model is matched to image structure in order to find possible instances of the model in the image. Matches between the relational model and image primitives are then used to determine probability distributions for the parameters of a geometric transformation. This transform maps a geometric model of the structure onto an instance in the image. This distribution may then be used to infer a probability map for pixel-based information such as edge responses. When combined with the original edge responses, an enhanced image is produce with more salient edge structure. Iteration of the procedure results in a consistent set of models and edge structure. The method is demonstrated on rectangles undergoing affine transforms.
\end{abstract}

\section{Introduction}

The Generalized Hough Transform is a method of recovering the parameters of a model from information such as edge positions in the image array. Although it is very effective in recovering model parameters, it has a number of drawbacks. The computational cost and storage requirements of the method rise rapidly as the number of model parameters increases, making it unsuitable for complex models. Since it's inception[5], many variants have been suggested to alleviate these problems. The randomised Hough transform(RHT) $[2,3]$ uses pairs of points to compute single entries in the parameter map. The probabilistic Hough transform[4] makes use of the fact that not all edge pixels must be processed to obtain an accurate model. Edge points are therefore sampled from the image array at random. Kalviainen and Hirvonen[1] exploit the connective structure of line points to improve the speed and accuracy of the method.

These methods may be viewed as encompassing a spectrum between the use of raw edge points and segmental entities such as connected line points. Although the use of more salient entities such as line segments increases the efficiency of the method, it renders it vulnerable to errors in the segmentation phase. It is our observation that image segmentation can be improved by utilising global models of possible structure, and these are exactly the kind of models produced by the parametric methods described above. Grimson[6,7] and Grimson and LozanoPérez $[8,9]$ have looked at the problem of interpreting segmental output, which 
may include noise and occulsion errors, by using global models of structure. They employ the interpretation tree[8] and a search algorithm to find matches between segmental structure and the model. This match is used to infer global tranformations of the $2 \mathrm{D}$ models.

Relational structure is a common and powerful way of representing the structure of objects in scenes. Methods of matching such structures to image segments have recently been developed which are tolerant to uncertainty and error $[14,10$, $11,15,13,12]$. The key element of this approach is a relational graph which describes the structure of an object by the relationship between sub-parts. This graph represents a model of the object. The image may then be segmented and their inter-relationships found in order to form a image graph. The task of object location is then one of finding a sub-graph isomorphism between the model and image graphs. Despite these advances, the power of such representations is often limited by the quality of the segmental output. It is the aim of this paper to couple the processes of model location and segmentation, with the aim of finding reliable pairs of mutually consistent arrangments of segmental structure and model parameters. Initially, the method is similar to that of Grimson in that it interprets segmentation in terms of a global structural model, which in our case is a relational model based on perceptual groupings of segments. However, once a putative relational model has been found, the correspondences are used to find possible transforms which map a geometric model onto the image. In fact, the accuracy of the segmentation is used to determine a probability distribution for the tranformation. This in turn allows us to establish a probability map for the location of edge structure in the image which can be used to enhance the original edge responses. Iteration of the method leads to the enhancement of salient edge structure while the remainder is discarded.

\section{Relational Model}

We begin with a description of the image, consisting of a set of segmental entities $V=\left\{v_{0}, v_{1}, \ldots, v_{n}\right\}$ representing, for example segments such as straight lines. These segments may be obtained by an edge detector followed by a standard edge polygonisation method. Each segment has a measurement vector associated with it which is derived from its properties in the image; thus for each segment $v_{i}$ there is a measurement vector $\boldsymbol{x}_{i}$. In the case of line segments this vector consists of the start and end points of the segment. Our task then is to determine any arrangements of these segments which are consistent with a relational model of the object of interest. A relational graph $G=\{V, E\}$ consists of a set of nodes $V=\left\{v_{0}, v_{1}, \ldots, v_{n}\right\}$, representing segments, and set of edges $E=\left\{e_{0}, e_{1}, \ldots\right\}$. Each edge $e_{i}=\left\{\left(v_{a}, v_{b}\right), \omega_{i}\right\}$ represents a perceptual relation between two segments, where $\omega_{i}$ is a label denoting the type of the relation. Such a graph can describe the mutual consistency constraints between segments in a compact way. These edges may either represent geometric relations such as Voronoi neighbours or perceptual structure such as colinearity or parallelism. Two such graphs $G_{0}, G_{1}$ are completely consistent when there exists a mapping between the node sets $\mathcal{M}: V_{0} \rightarrow V_{1}$ which is one-to-one and is such that 
the mapped edges have the same labels. In a scene there are many objects and resulting segments. The task of locating structure consistent with a particular object model becomes one of mapping the relational graph for the object onto a sub-graph of the scene segments. Depending upon the complexity of the object model, missing and spurious segments may also become an issue, and inexact sub-graph mapping may be required. There are many algorithms available in the literature to achieve this end $[14,10,11,15,13,12]$.In this paper we have used the method described in Wilson and Hancock [15].

\subsection{Affine Rectangle Model}

In the experiments conducted in section 5, we use an affine rectangle model. The relational part of this model is shown in figure 1 .

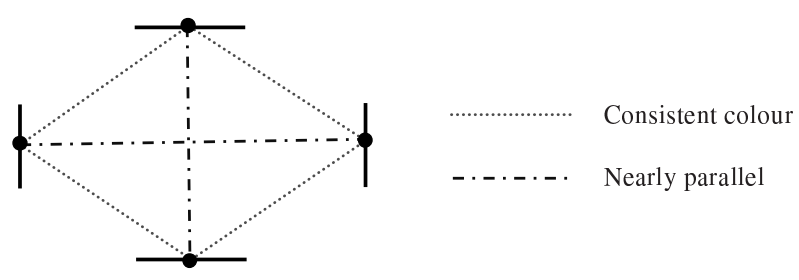

Fig. 1. Model of an affine rectangle

The nodes of this graph are straight line segments which represent the edges of the rectangle. We do not employ a fully perspective model of the rectangle because such a model is too unconstrained, since any four lines will make such a rectangle. Instead, we confine our attention to the set of rectangles whose projections are nearly affine, i.e. whose opposite sides are nearly parallel. Our first graph relation is therefore the 'parallel' relation between line segments. The other property of a consistent rectangle is that the interior of the rectangle is one colour. The second relation in the graph connects edges which have similar colours on the interal side of the edge they represent. In other words, they represent the constraint that the interior of the rectangle must be the same colour. We also exclude very narrow parallelograms with small corner angles.

\section{Geometric Model}

Once a mapping between an object model and a sub-graph in the scene has been located, the co-incident segments provide information about the geometry of the object. In particular, they may be used to infer a transform between the object model and it's realisation in the scene. In the case of our affine rectangle model, the affine mapping between the canonical model of a unit square and image is found by associating each one of the sides with a line segment identified by a match to the relational model. We can then use this transform to infer where the edges should be in the image, and enhance the edge responses at these locations. 
However, there are some problems with using a precise geometric model for projection in this way. There are likely to be errors in the positions of the image segments and, due to discrete nature of the image, edge structure may not lie precisely on the line specified by the transformed geometric model. In order to successfully locate the edge structure, we need to take account of the uncertainty in the position of line segments and therefore in the affine mapping. The analysis of the appropriate probability distributions is very complex, even under simple models such as the affine transformation. Instead, we adopt an approach based on finding the first order variations of segment parameters and then adopt a normal distribution of transform parameters. We commence by looking at the errors in line segments extracted from the image.

\subsection{Errors in Segments}

For each segment in the image, we can calculate not only the segment measurement parameters, but also the parameter variances using a least-squares approach. For example in the case of a segment containing the points $\left\{\left(x_{i}, y_{i}\right), i=\right.$ $0 \ldots n\}$ and a straight line segment model, the variances are given by, for a mainly horizontal line,

$$
\begin{aligned}
\operatorname{var}(m) & =\frac{\sum\left(y_{i}-m x_{i}-c\right)^{2}}{n \sum x_{i}^{2}-\left(\sum x_{i}\right)^{2} / n} \\
\operatorname{var}(c) & =\frac{\sum\left(y_{i}-m x_{i}-c\right)^{2}}{n-\left(\sum x_{i}\right)^{2} / \sum x_{i}^{2}} \\
\operatorname{cov}(m, c) & =-\frac{\sum\left(y_{i}-m x_{i}-c\right)^{2} \cdot \sum x_{i}}{n^{2} \sum x_{i}^{2}-\left(\sum x_{i}\right)^{2}}
\end{aligned}
$$

The variance in the centre-points of the segments is $\operatorname{var}\left(y_{c}\right)=\sum\left(y_{i}-m x_{i}-\right.$ $c)^{2} / n$. If the line is mainly vertical, the roles of the $x$ and $y$ points must be reversed.

\subsection{Affine Rectangle Model}

The geometric model of the rectangle consists of the four corner points and four lines joining the corner points (figure 2).

The segments are straight lines, and therefore the errors in the segment parameters are those given in equation 1 . However, because of the affine transformation, we require the four corner points to define the transform. We are therefore interested in the errors in the crossing points of the line segments which make up the rectangle. These are given by equation 2 where corner $n$ is formed by the intersection of lines $a$ and $b$.

$$
\begin{aligned}
\operatorname{var}\left(x_{n}\right)=v_{n}= & x_{n}^{2}\left[\frac{\operatorname{var}\left(c_{a}\right)+\operatorname{var}\left(c_{b}\right)}{\left(c_{a}-c_{b}\right)^{2}}+\frac{\operatorname{var}\left(m_{a}\right)+\operatorname{var}\left(m_{b}\right)}{\left(m_{a}-m_{b}\right)^{2}}\right] \\
\operatorname{var}\left(y_{n}\right)=w_{n}= & y_{n}^{2}\left[\frac{c_{b}^{2} \operatorname{var}\left(m_{a}\right)+c_{a}^{2} \operatorname{var}\left(m_{b}\right)+m_{a}^{2} \operatorname{var}\left(c_{b}\right)+m_{b}^{2} \operatorname{var}\left(c_{a}\right)}{\left(m_{a} c_{b}-c_{a} m_{b}\right)^{2}}\right. \\
& \left.+\frac{\operatorname{var}\left(m_{a}\right)+\operatorname{var}\left(m_{b}\right)}{\left(m_{a}-m_{b}\right)^{2}}\right]
\end{aligned}
$$




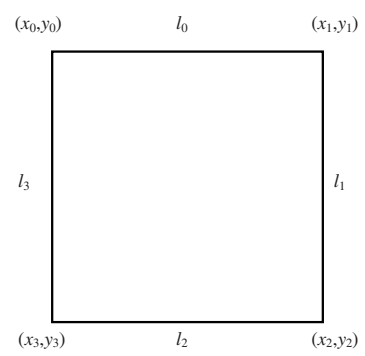

Fig. 2. The geometric model of the rectangle

By using a normal distribution and the variance provided by (2), the probability of a transform $G$ which maps the square onto the endpoints $\left(x_{0}, y_{0}\right) \ldots\left(x_{3}, y_{3}\right)$ is given by

$$
P\left(G \mid \mathcal{M}^{*}, \mathcal{L}\right)=\frac{1}{2 \pi \sqrt{\prod_{i} v_{i} w_{i}}} \exp \left[-\sum_{i}\left(x_{i}-\bar{x}_{i}\right)^{2} / 2 v_{i}+\left(y_{i}-\bar{y}_{i}\right)^{2} / 2 w_{i}\right]
$$

Here $\mathcal{M}^{*}$ represents the match found between image and relational model, and $\mathcal{L}$ represents the edge pixels involved in the lines which make up the model.

\section{Enhancing Contours}

Our final aim is to iteratively re-compute the edge probability maps, image segments and relational mappings to find a consistent description of the model and scene. To achieve this aim, we exploit a relaxation method[16] which makes successive approximations to the MAP estimate of the features and labels.

We begin by expanding over the set of possible transformations $G$ and factorising the probability model:

$$
P\left(F, \mathcal{M}^{*}, \mathcal{L}\right)=\sum_{G} P\left(F, \mathcal{M}^{*}, \mathcal{L}\right)=P(F) \sum_{G} P\left(G \mid \mathcal{M}^{*}, \mathcal{L}, F\right) P\left(\mathcal{M}^{*}, \mathcal{L} \mid F\right)
$$

Here $F$ is the field of edge responses. The tranform probability may be considered to be conditionally independent of the feature space given a particular match and set of line segments, i.e. $P\left(G \mid \mathcal{M}^{*}, \mathcal{L}, F\right)=P\left(G \mid \mathcal{M}^{*}, \mathcal{L}\right)$. The quantity $P\left(\mathcal{M}^{*}, \mathcal{L} \mid F\right)$ is precisely that which we optimise to find the best match between relational model and image. Our iterative relaxation algorithm is then specified by

$$
P^{(n+1)}(F)=P^{(n)}(F) P\left(\mathcal{M}^{*}, \mathcal{L} \mid F\right) \sum_{G} P\left(G \mid \mathcal{M}^{*}, \mathcal{L}\right)
$$

\section{Results}

In the first set of experiments, we apply the technique to a synthetic image of a quadrilateral. The image is shown in figure 3. As it stands, this is a trivial 

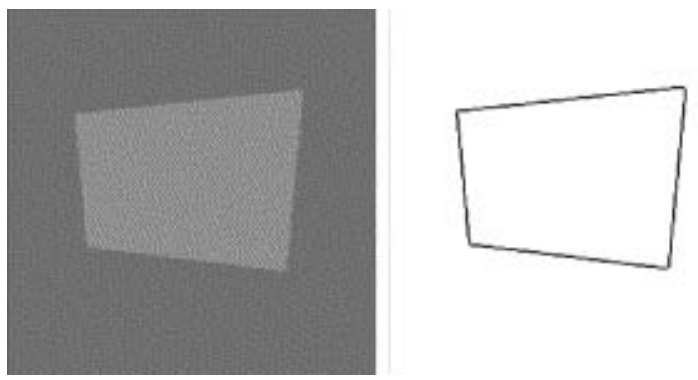

Fig. 3. Left: Quadrilateral image, Right: Result of edge detection

problem since the initial edge configuration is very good. The results of edge detection are also shown in figure 3. In order to provide a challenging task, we have added various levels of Gaussian noise to the image in figure 3. The new images have signal to noise ratios of $2,1,0.5$ and 0.25 respectively. The final image therefore has noise with standard deviation four times the size of the edge step. Figure 4 shows the results of applying our method; the image in the left hand column is the original image; the central column is the edge detection result, and the image in the right hand column represents the final edge configuration after application of the algorithm. The method is able to accurately reconstruct the correct edge configuration even under very high levels of noise.

Figure 5 shows these results quantitatively. Here we have used a pixel based measure of accuracy based on average minimum distance. If $P=\left\{p_{0}, p_{1}, \ldots, p_{m}\right\}$ is the set of pixels in a perfect edge configuration, and $Q=\left\{q_{0}, q_{1}, \ldots, q_{n}\right\}$ is the set of pixels in the test image, the forward error $e_{f}$ is given by $e_{f}=$ $\frac{1}{m} \sum_{i=0}^{m} \min _{j} d\left(p_{i}, q_{j}\right)$, where $d($.$) is the Euclidean distance. This error measures$ both misplaced and missing structure in the test image. The backwards error is given by $e_{b}=\frac{1}{n} \sum_{i=0}^{n} \min _{j} d\left(q_{i}, p_{j}\right)$ and measures misplaced and extra spurious structure. By combining these measures in $e=\left(e_{f}+e_{b}\right) / 2$, we obtain a measure which reflects misplaced, spurious and missing edge structure. When $e=0$, the configurations are identical. The signal level is 16 . In this simple case, our model can accurately reconstruct the edge configuration even in the presence of extreme noise because of the global model of structure.

In the second set of experiments, we add a set of background non-quadrilateral distractors. These distractors both disrupt the initial edge configuration and test the algorithms ability to discard irrelevant structure. The initial image is shown in figure 6. Again we add Gaussian noise to the image and attempt to reconstruct the original rectangle. The results are shown in figure 6(right).In this case, although the original configurations are worse, the final result is superior because the background contrast is increased by the distractors.

In the third set of experiments, we apply the quadrilateral model to an scene from an office desk. There are a number of prominent quadrilaterals present in the image, and a considerable amount of clutter, including some non-rectangular objects and background texture. Figure 7 shows the original image and the resulting edge strengths and edge segmentation. 

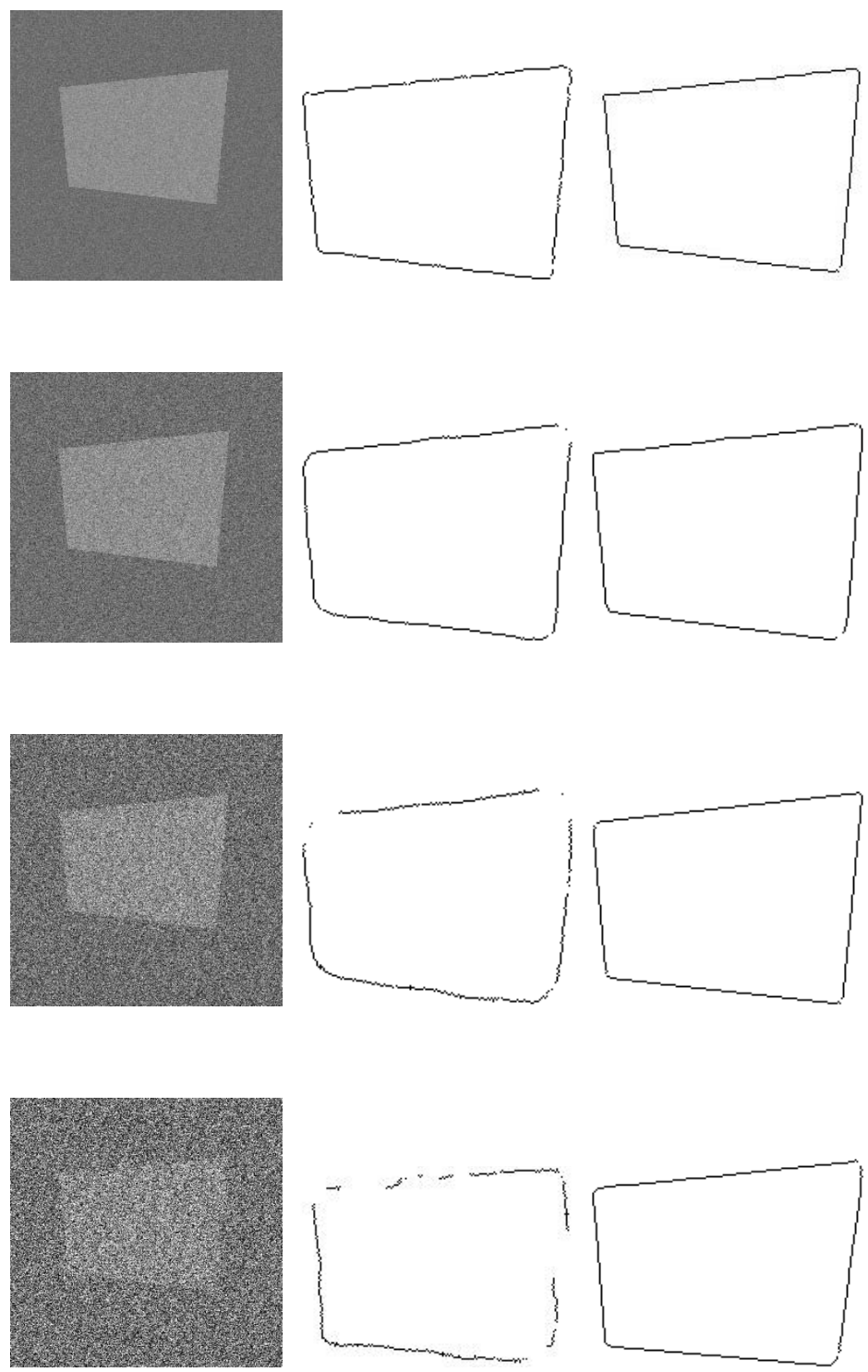

Fig. 4. Left column: original image; Central column: original edge detection results; Right column: final edge configuration

The final image is the result from the tenth and final iteration. Both the background and non-rectangular objects have been eliminated. However, some spurious linear structures remain, resulting from the participation of some edges in multiple quadrilaterals.

This experiment reveals some important points about the method and the quadrilateral model. Firstly, gaps in the edges can only be closed where some 


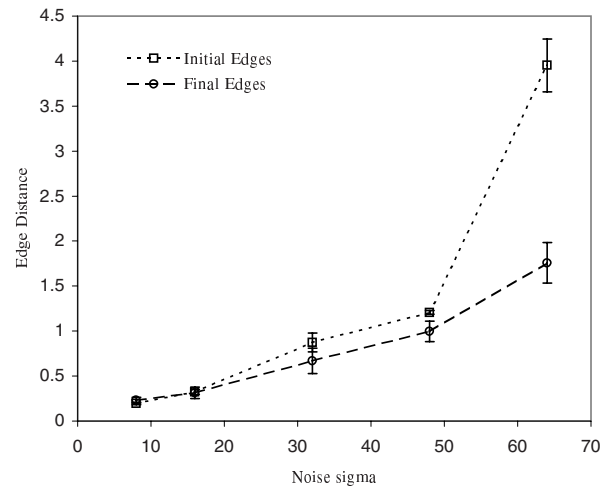

Fig. 5. Performance of method on simple rectangle image
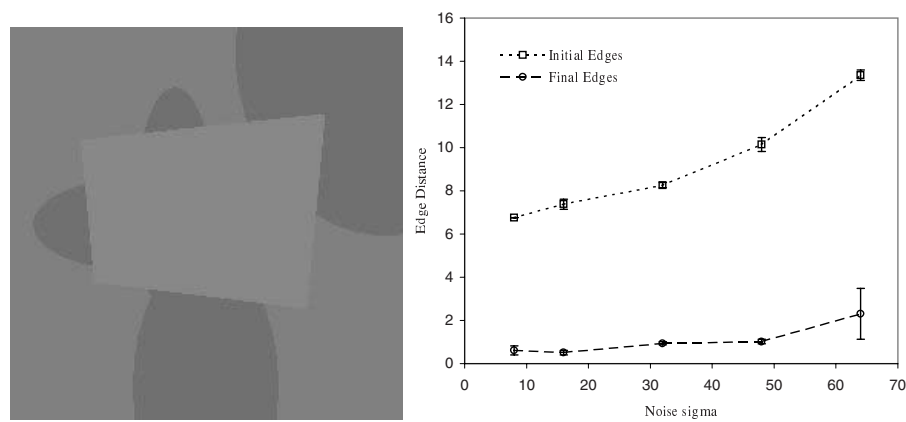

Fig. 6. Left: original distractors image; Right: Performance of method at different noise levels
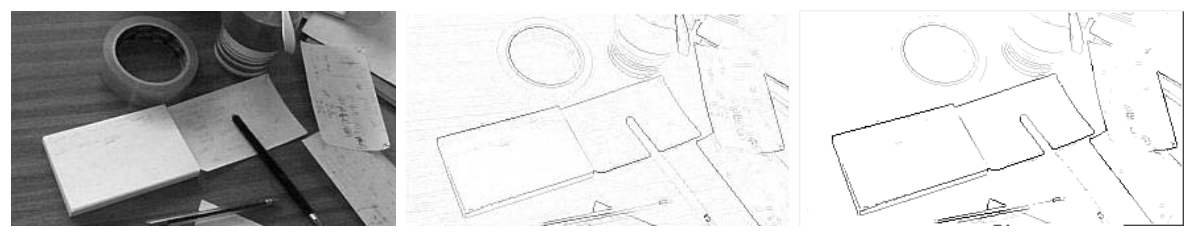

Fig. 7. The original image (left), the initial edge field (centre) and the final edge field (right)

evidence exists in the responses of the edge detector. For example, where the pen crosses the notepad, no evidence for the notepad edge exists. Secondly, the quadrilateral model is limited in the sense that the four edges may appear anywhere in the image, and so if one edge is missing, it becomes impossible to reconstruct the model. We intend to address these issues in future work by constructing a more sophisticated model of feature probability and employing more sophisticated perceptual groups. 


\section{References}

1. H. Kalviainen and P. Hirvonen, "An Extention to the Randomized Hough Transform", Pattern Recognition Letters,18:1 pp 77-85 1997.

2. L. Xu and E. Oja and P. Kultanen, "A new curve detection method - Randomized Hough Transform(RHT)", Pattern Recognition Letters,11:5 pp 331-338 1990.

3. L. Xu and E. Oja, "Randomized Hough Transform(RHT) - Basic mechanisms, algorithms and computational complexities", CVGIP: Image understanding, 57:2 pp 131-154 1993.

4. J. R. Bergen and H. Shvaytser, "A probabilistic algorithm for computing Hough Transforms", Journal of Algorithms, 12:4 pp 639-656 1991.

5. R. O. Duda and P. E. Hart, "Pattern Recognition and Scene Analysis", Wiley, New York 1973

6. W. E. L. Grimson, "On the Recognition of Curved Objects", IEEE Transactions on Pattern Analysis and Machine Intelligence, 11:6 pp632-643 1989

7. W. E. L. Grimson, "On the Recognition of Parameterized 2D Objects", International Journal of Computer Vision, 2:4 pp353-372 1989

8. W. E. L. Grimson and T. Lozano-Pérez, "Localizing overlapping parts by searching the interpretation tree", IEEE Transactions on Pattern Analysis and Machine Intelligence, 9:4 pp469-482 1987

9. W. E. L. Grimson and T. Lozano-Pérez, "Model-Based recognition and localization from sparse range or tactile data", International Journal of Robotics Research, 3:3 pp3-35 1984

10. K. Boyer and A. Kak, "Structural Stereopsis for 3D vision", IEEE Transactions on Pattern Analysis and Machine Intelligence, vol. 10, pp 144-166, 1998

11. W. Christmas and J. Kittler and M. Petrou, "Structural Matching in Computer Vision using Probabilistic Relaxation", IEEE Transactions on Pattern Analysis and Machine Intelligence, vol. 17, pp 749-764, 1995

12. B. T. Messmer and H. Bunke, "Efficient Error-Tolerant Subgraph Isomorphism Detection", Shape, Structure and Pattern Recognition, pp 231-240, 1994

13. A. Sanfeliu and K. S. Fu, "A Distance Measure Between Attributed Relational Graphs", IEEE Systems, Man and Cybernetics, vol. 13, pp 353-362, 1983

14. L. Shapiro and R. M. Haralick, "Structural descriptions and inexact matching", IEEE Transactions on Pattern Analysis and Machine Intelligence, vol. 3, pp 504519,1981

15. R. C. Wilson and E. R. Hancock, "Structural Matching by Discrete Relaxation", IEEE Transactions on Pattern Analysis and Machine Intelligence, 19:6, pp 634648, 1997

16. J. Kittler and E. R. Hancock, "Combining evidence in Probabilistic Relaxation", International Journal of Pattern Recognition and Artificial Intelligence 3, pp29-52, 1989 